Fig. 3. Neurogliazelle nach 5 und 7 Stunden.

Fig. 4, 5, 6. Ganglienzellen des Gehirns nach 7 Stunden.

Fig. 7, 8, 9, 10, 11, 12. Neurogliazellen des Hirns nach 12 Stunden.

Fig. 13. Purkinje'sche Zelle nach 1 Stunde.

Fig. 14, 15, 16, 17. Ganglienzellen des Hirns nach 1 Stunde.

Fig. 18, 19, 20. Ganglienzellen des Hirns nach 7 Stunden.

Fig. 21. Purkinje'sche. Zelle nach 7 Stunden.

Fig. 22, 23, 24. Ganglienzellen des Hirns nach 12 Stunden.'

Fig. 25, 26, 27. Ganglienzellen des Hirns nach 24 Stunden.

Fig. 28. Kerne der Nervenzellen des Birns nach 24 Stúnden.

Fig. 29, 30, 31, 32, 33. Ganglienzellen des Rückenmarkes nach 1 Stunde.

Fig. 34, 35, 36, 37, 38. Ganglienzellen des Rückenmarkes nach 7 Stunden.

Fig. 39, 40. Ganglienzellen des Rückenmarkés nach 24 Stunden.

\title{
XXIII.
}

\section{Beiträge zur Kenntniss der Lymphcirculation in der Grosshirnrinde.}

(Aus dem Laboratorium der psychiatrischen Klinik in Jena.)

Von Prof. O. Binswanger und Dr. H. Berger.

(Hierzu Taf. X-XI.).

Trotz der Arbeiten von His, Key und Retzius, Obersteiner, Bevan Lewis u. A. gehört die Frage nach der Säftebewegung im centralen Nervensystem zu denjenigen, auf die nach dem heutigen Stande unserer Kenntnisse keine sichere Antwort gegeben werden kann. Am verbreitesten ist wohl diejenige Ansicht, welche Obersteiner der Darstellung der Lymphbahnen in seinem bekannten Werke über den Bau der nervösen Centralorgane zu Grunde legt, die wir hier kurz recapituliren wollen, indem wir uns nur auf die Verhältnisse des Grosshirns und speciell der Rinde beschränken. Die Rindengefässe sind von einem doppelten Lymphraum, einem intraadventitiellen - zwischen Adventitia und Muscularis gelegenen, dem Vir chow - Ro b i n'schen Raum oder dem adventitiellen Lymphraum 


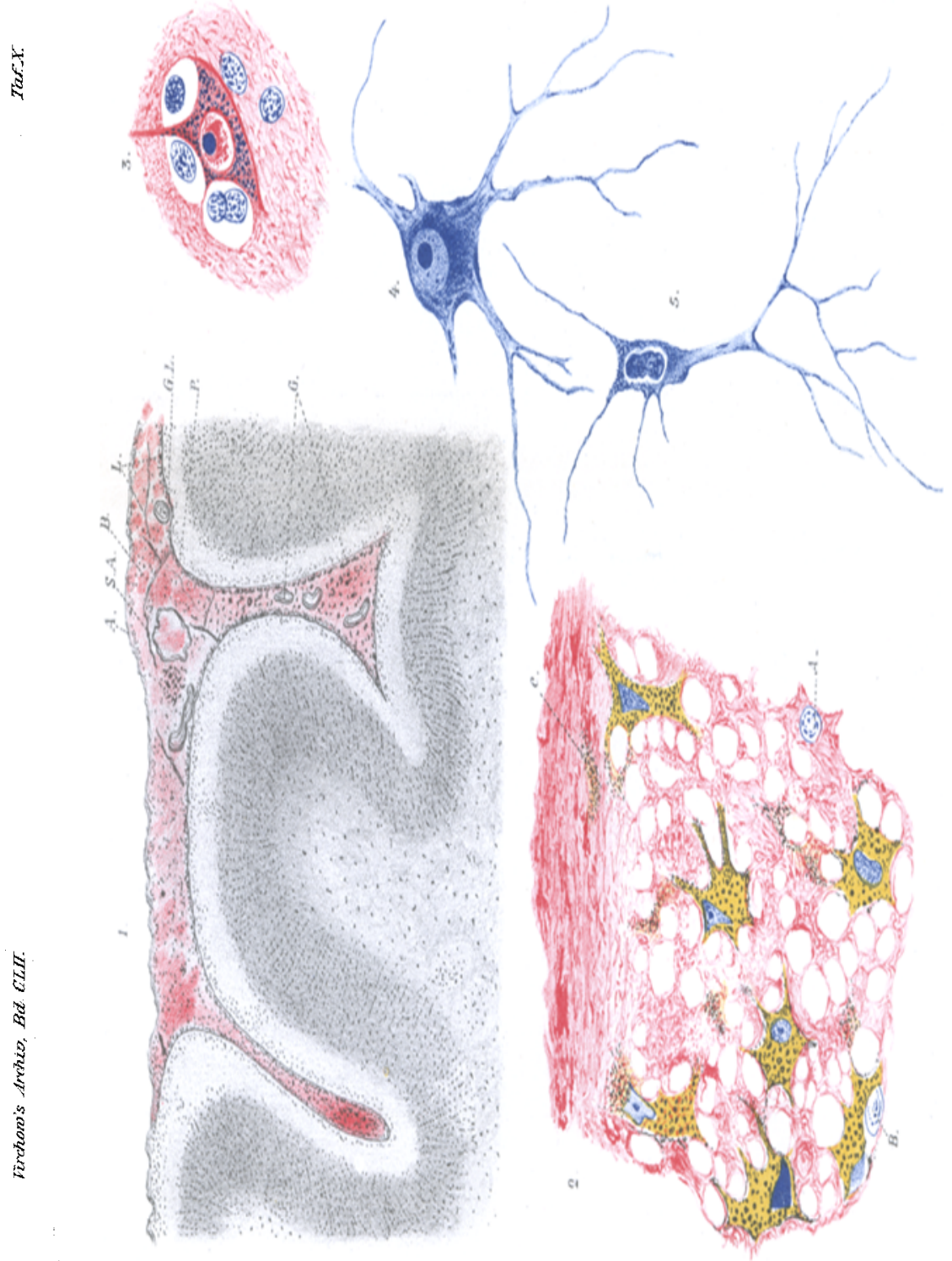


है
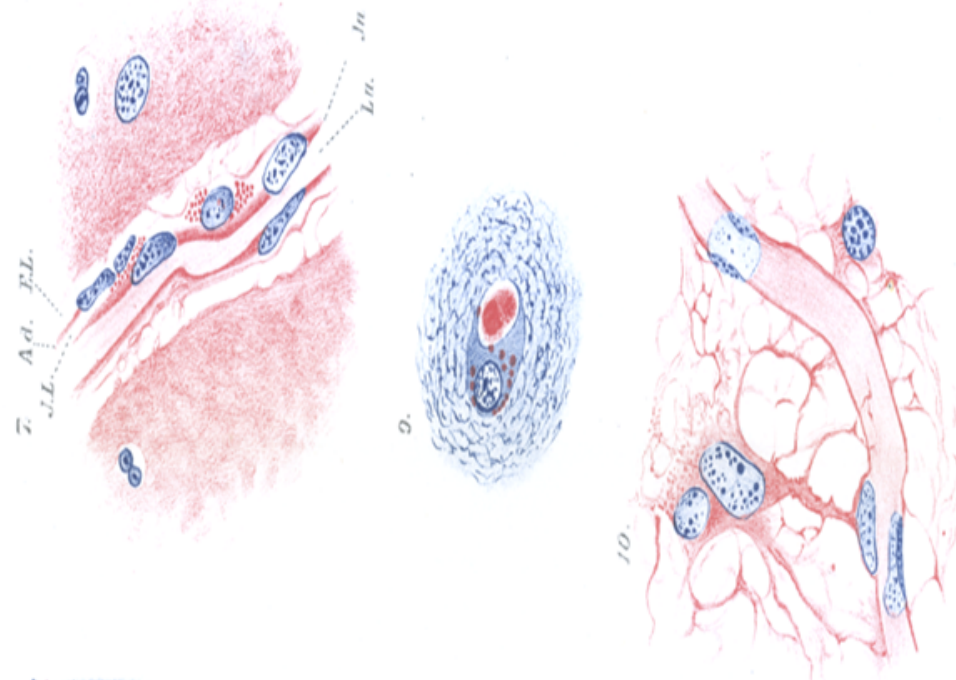

है
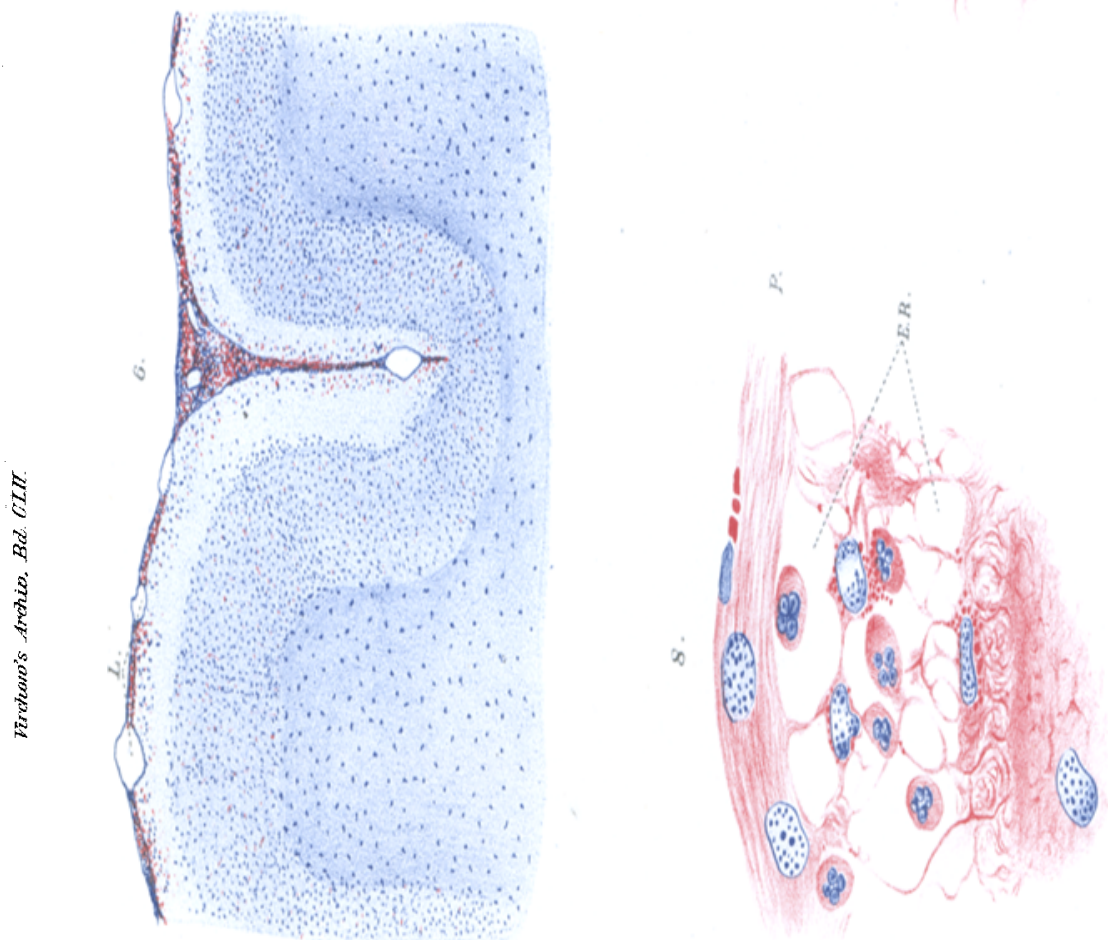
s. str. - und einem extraadventitiellen, dem His'schen") oder perivasculären Lymphraum s. str. umgeben; zwischen beiden Lymphräumen besteht nirgends eine offene Communication, sondern jedẹr derselben hängt mit einem geschlossenen System von Lymphräumen zusammen. Der extraadventitielle Lymphraum entsendet einerseits dünne Ausläufer, die sich in der Nähe der Ganglienzellen zu den pericellulären Räumen, an deren Existenz nicht mehr gezweifelt werden kann, erweitern, andererseits geht er peripheriewärts direct in das Lacunensystem des Epicerebralraums über und communicirt auch mit den Arnold'schen Lymphbahnen der Pia; das zweite Lymphsystem bildet der intraadventitielle Lymphraum, der mit dem Subarachnoidalraum in freier Communication steht. Auf den specielleren anatomischen Bau dieser Lymphsysteme einzugehen, scheint uns hier nicht nöthig, jedoch muss erwähnt werden, dass Kronthal in der Grosshirnrinde eigene Lymphgefässe nachgewiesen und dass Riedel Communicationsröhren zwischen den intraadventitiellen Lymphräumen verschiedener Gefässe beschrieben hat. Dass die subarachnoidalen Räume in freier Communication mit dem Ventrikelsystem stehen, ist eine längst bekannte Thatsache, und wird hier nur mit Rücksicht auf den unten zu beschreibenden Obductionsbefund nochmals hervorgehoben.

Folgende klinische Beobachtung gab uns Veranlassung, der dunklen Frage der Saftbahncirculation in der Grosshirnrinde von Neuem näher zu treten.

Der 54 Jahre alte Arbeiter A. P. wurde am 13. Juli 1897 in die hiesige psychiatrische Klinik eingeliefert. Aus der Anamnese ist erwähnenswerth, dass Patient erblich nicht belastet ist, und immer als intelligenter und fleissiger Arbeiter galt. Vor 2 Jahren wurden ihm die linksseitigen Axillardrüsen entfernt, da sie krebsig entartet waren. Patient war dann vollständig gesund bis vor 6 Wochen, wo er plötzlich mit heftigen Schmerzen im Rücken erkrankte, bald zeigte sich, dass Patient theilweise seine Umgebung verkannte, sich bei der Arbeit wähnte u. s. w. Er wurde daher von dem Arzte der Anstalt zugeschickt. Patient ist von mittlerer Grösse mit gut entwickelter Musculatur. Der Schädel ist brachycephal, es besteht eine geringe rechtsseitige Stenose, er zeigt keinerlei Narben oder frisehe Verletzungen.

1) W. His, Ueber ein perivasculäres Kanalsystem in den nervösen Centralorganen und über dessen Beziehungen zum Lymphsystem. Zeitschr. für wissenschaftl. Zool. Bd. XV. S. 127. 
Die Pupillen sind beide verzogen, die linke ist vollständig lichtstarr, die rechte reagirt träge. Es besteht ein sehr starker Tremor aller Muskeln, keine Ataxie, aber eine leichte Parese des linken Beines. Knie- und Acbillessehnenphänomene sind nicht erhältlich. Die Kopfpercussion ist sehr schmerzhaft, und zwar an allen Stellen des Schädels. Ophthalmoskopisch findet sich eine stark entwickelte, beiderseitige Staungspapille. Die Sprache ist leicht băsitirend, der Gesichtsausdruck ängstlich. Das Gebör ist ebenso wie der Geruch intact. Patient ist über Ort und Zeit ziemlich gut orientirt, rechnet einfache Exempel, wenn auch langsam, so doch richtig aus, er muss sich bisweilen lange auf Antworten besinnen. In den folgenden Tagen zeigt Patient zunehmende Erregung, er spricht fortwährend incohärent laut vor sich hin, steigt ausser Bett, drängt zur Thüre binaus. Patient verkennt seine Umgebung, glaubt sich bald daheim, bald bei der Arbeit. In der Folge knirscht er bäuffig und lange mit den Zäbnen; der Tremor der gesammten willkürlichen Musculatur nimmt so an Intensität zu, dass jede active Muskelbewegung unmöglich wird. Die Symptome gehen zeitweise zurück, und Patient zeigt dann eine deutliche sensorische Aphasie, und klagt viel über Kopfschmerzen. Bald trat jedoch wieder eine zunehmende Verschlimmerung ein, es zeigten sich klonische Zuckungen im linken Mundfacialis und eine Hyperästhesie des ganzen Körpers unter leichten Fieberbewegungen. Patient wurde comatös, konnte nicht mehr schlucken, es zeigt sich eine conjugirte Deviation der Augen nach rechts oben mit Strabismus divergens. Der Tod erfolgte unter hohen Temperatursteigerungen, in Folge des eintretenden Lungenödems.

Bei der 3 Stunden nach dem Tode vorgenommenen Section (Geh.-Rath Prof. Müller) fand sich eine graue Degeneration der Seiten - und Hinterstränge des Rückenmarks. Die Dura des Gehirns ist von der Glastafel leicht abhebbar, dieselbe ist von mittlerer Dicke, ihre Innenfläche ist bleich und glatt. Im Längsblutleiter ist flüssiges Blut. Die Arachnoides ist zart, desgleichen die Pia, in deren Maschen findet sic klare, stellenweise sanguinolente Flüssigkeit. Die Stirnwindungen sind leicht gelblich verfärbt. Die vordere linke Centralwindung ist in ibrer Mitte dureh eine rundliche, röthlich-graue, $10 \mathrm{~mm}$ im Durchmesser messende Neubildung flach vorgebuchtet. Das rechte laterale Scheitelläppchen zeigt an seinem vorderen Ende eine gleich grosse, grauweisse, flache Vorbuchtung. Die Dura basalis ist normal. Im linken Querblutleiter findet sich an der Umbiegungsstelle in den senkrechten Verlauf ein gelblicher, festhaftender Thrombus.

Das Gebirngewicht beträgt $1287 \mathrm{~g}$.

Basale Meningen im Ganzen zart, über beiden Schläfenlappen stellenweise rothgelb verfärbt, gelbliche Färbung des Chiasma, beider Oculomotorii; Abducentes und Olfactorii.

Die Hirnstiele sind bleich, der Aquäduct ist erweitert, ebenso ist die IV. Kanmer erweitert, ihr Ependym grob granulirt, schmutzig - gelb verfärbt.

Das Kleinhirn ist mässig fest. 
Das Ependym des III. Ventrikels und die Oberfläche des Balkens ist gelblich verfärbt. In den Seitenkammern findet sich rechterseits ein umfänglicher Klumpen schwarzbraunen bis bräunlich-gelben, geronnenen Blutes neben flüssigem Blut.

Das in der Stria cornea verlaufende Gefäss, ișt an der Uebergangsstelle des Körpers des Nucl. caudat. in den Schwanz an umschriebener Stelle blossliegend, das überliegende Ependym im Umfang eines 10-Pfennigstücks zerstört durch einen Erguss theils flüssigen, theils geronnenen Blutes.

Das rechte Grosshirn ist bleich, mässig fest, weisse und graue Substanz wohl gesondert.

Die Stammganglien sind bleich. Hinterhorn erweitert, Plexus chorioideus leicht ödematös.

Die schon oben erwähnte Neubildung im rechten oberen Scheitelläppchen ist auf dem Durchschnitt rötblich-grau, und reicht bis zur Pia.

Die linke Hemisphäre ist mässig fest, die weisse Substanz weiss, die graue gut abgesetzt, blass - grau, der oberste Theil des Balkens ist leicht gelblich gefärbt.

Das Ependym der Seitenkammern ist weniger gelb als rechts. terirt.

Plexus mässig ödematös, die hintere Hälfte des Hinterhorns ist obli-

Die linke vordere Centralwindung ist in ibrer Mitte von einem, nach aussen überragenden, erbsengrossen, röthlich-grauen Neubildungsknoten eingenommen; unter der Neubildung findet sich eine kirschengrosse, mit dünner bräunlich - gelber Wand, und dickem, chocoladebraunen Inhalt versehene Elöhle.

Die Section der übrigen Körperhöhlen ergiebt: Oedema pulmonum, Thrombus im linken Lungenarterienast.

Spindelförmige Erweiterung der Carotis interna dextra am Ursprung.

Mässige Arteriosklerose.

Vereinzelte Kystome der Niere.

In Uebrigen bietet der Befund keine Besonderbeiten dar.

An dem in 10 procentiger Formalinlösung gehärteten Gehirn erkennt man deutlich die sanguinolente Verfärbung der in den Piamaschen enthaltenen Flüssigkeit. An der rechten Hemisphäre ist diese subarachnoidale Blutung am intensivsten über den Temporallappen, dem unteren Theil des Stirnlappens und des unteren Parietallappens; die Intensität nimmt nach der Mantelkante zu ab, die Blutung geht aber noch auf den Lobus paracentralis und den Präcuneus über. Die linke Hemisphäre zeigt die intensivste Verfärbung über dem lateralen Theil des Occipitallappens, über dem Lobus parietalis inferior, den daran anstossenden Theil des Temporallappens und der orbitalen Fläche des Stirnlappens. 
An den den verschiedensten Rindenstellen entnommenen, in Alkohol nachgehärteten, in Paraffin eingebetteten, und mit Thionin, bezw. Hämatoxylin gefärbten Präparaten zeigt sich, dass entsprechend den verfärbten Stellen der Oberfläche sich ein mehr oder minder starker, subarachnoidaler Bluterguss findet (vergl. Fig. 1), der nur vom Ventrikel aus dahin gelangt sein kann. Die Arachnoides ist an einzelnen Stellen durch den Bluterguss bis zu einer. Entfernung von $8 \mathrm{~mm}$ von der Pia gehoben, der mikroskopische Befund, der frische, rothe Blutkörperchen neben altem schollenförmigen Blutfarbstoff, der zum Theil in Leukocyten eingeschlossen ist, zeigt, deutet auf eine wiederholte Blutung aus dem Aneurysma der Arteria striae corneae hin. Die Dicke der subarachnoidalen Blutmassen ist am grössten in dev Furchentiefen und den Stellen zwischen den Oberflächen der Windungen. Alle Pia-Gefässe sind strotzend gefüllt. An an. deren Stellen liegen Pia und Arachnoides eng an einander, und es findet sich keine Spur von Blut oder Fárbstoff zwischen beiden Häuten. Die oben genannten Tumoren ergaben sich bei der mikroskopischen Untersuchung als ächte Carcinomknoten. Indem wir hier auf die höchst interessante und lohnende Gegen: überstellung des Obductionsbefundes und der klinischen Beobachtung verzichten, wollen ${ }^{1}$ ) wir uns nur mit den auf die Erage

1) Nur auf die ätiologisch - Elinische Bedeutung des Falles wollen wit kurz hinweisen. Bekanntlich ist im Laufe der letzten Jabrzebnte dif Syphilis mehr und mehr als die hauptsäcblichste Quelle der Dementic paralytica erkannt worden. Neuere Untersucher, vor Allem KrafftE bing und seine Schüler, sind sogar so weit gegangen, die Paralyst für eine ausschliesslich syphilitische Krankbeit anzusprechen. Diest Anschaung ist sicher zu weitgehend.' Wenn die Syphilis auch eine, uno wahrseheinlich die hauptsächlichste Ursache einer degenerativen Erkrankung des Centralnervensystems ist, so können. sicher auch andere gewebsscbädigende Einflüsse diese Krankheit herbeiführen. Hier lieg1 eine reine Beobachtung vor, in welcher bei dem Mangel jeder syphiliti. schen Antecedentien die carcinomatöse Erkrankung den paralytischer Prozess verursacht hat. Heredität, Alcoholismus und Trauma können ebenfalls ausgeschlossen werden, dagegen ist die Arteriosklerose, die dem fortgeschrittenen Lebensalter entspricht, wahrscheinlich mit verantwortlich zu machen für die Ernährungsstörung des Gehirns; durch sie wurde der Boden bereitet, auf dem die bislang unbekannten Toxine deI Carcinose diesen degenerativen Einfluss ausüben konnten. 
der Lymphcirculation sich beziehenden Befunden beschäftigen. Es hatte hier zweifellos ein wiederholter Erguss von Blut in die Seitenkammern stattgefunden, und das Blut war von da in die subarachnoidalen Räume gelangt. Das weitere Schicksal dieses Blutes ist es, das uns hier interessiren soll. Bei stärkerer Vergrösserung, und namentlich bei Anwendung der Oelimmersion zeigte sich, dass die in Folge des paralytischen Krankheitsprozesses deutlicher hervortretenden und wohl auch an Zahl vermehrten Gliazellen der Molecularschicht der Rinde massenhaft von feinkörnigem Blutpigment erfüllt waren (vergl. Fig. 2). Die Gliazellen selbst waren stark gequollen und traten schon bei schwacher Vergrösserung sehr deatlich in dem vielfach zerklüfteten, mit Eosin schwach rothgefärbten Grundgewebe hervor. Die in die Rinde einstrahlenden Gefässe sind strotzend gefüllt, auch an ihnen findet sich innerhalb des intraadventitiellen Lymphraumes feinkörniges Blutpigment theils frei, theils an Zellen gebunden. Bei der Oelimmersion (Apochrom. 2,0 mm und Comp.Oc. 4) zeigen die Gliazellen der Molecularschicht an den Stellen des subarachnoidalen Blutergusses eine vollständige Anfüllung des gequollenen und scharf hervortretenden Zellleibs mit goldgelbem, feinkörnigem Blutpigment. Diese Anfüllung mit Pigment nimmt nach der Tiefe, d. h. der Schicht der kleinen Pyramidenzellen zu rasch an Intensität $a b$, so dass keine pigmenthaltige Zelle den obersten Theil der kleinen Pyramidenzellen erreicht. Auch die Gliahülle zeigt in ihren Zellen sehr reichliches Pigment, die Ausläufer einzelner dieser Zellen, die prall mit feinen Pigmentkörnchen angefüllt sind, können weithin verfolgt werden und enden an der Oberfläche des Gehirns. Die Gliazellen sind beträchtlich vergrössert und zeigen einen Durchmesser von durchschnittlich $20 \mu$. Der Zellleib zeigt eine deutliche, netzfôrmige Struktur, und in diese Netze sind die feinen Blutpigmentkörnchen eingelagert. Die zahlreichen Ausläufer der Zellen sind stark verbreitert und überall mit den gleichen Blutresten erfüllt. An manchen Stellen findet man gelblich gefärbte Stränge von gleicher Beschaffenheit (vergl. Fig. 2, C), welche keine andere Deutung zulassen, als dass sie abgetrennte Gliaausläufer sind, deren zugehörige Zellen in anderen Schnittniveaus liegen. Die Gliakerne dieser pigmenthaltigen Zellen 
sind meist in unförmige (bald eckige, bald wurstförmige, bald dreizipflich ausgezogene), mit Thionin tief dunkelblau gefärbte Gebilde umgewandelt. Während an den normalen Gliakernen die charakteristische Kernstruktur der Gliazellen sich immer nachweisen lässt, kann man an diesen deformirten, gleichmässig dunkel gefärbten und meist verkleinerten Kernen keinerlei Details unterscheiden. Die Kerne sind bald im Centrum, bald an der Peripherie der Zelle gelegen und man kann sich des Eindrucks nicht erwehren, als seien sie im letzteren Fall von dem massenhaft aufgenommenen Blutpigment an die Wand gedrückt. Neben diesen pigmenthaltigen Gliazellen finden sich in denselben Schnitten solche, die ganz den gewöhnlichen Typus der normalen Gliazellen; bei denen man meistens nur den Kern sieht, repräsentiren; diese letzteren zeigen keine Spur von Blutpigment in ihrem Innern und die Kerne besitzen die so typische Kernstruktur der Gliazellen (vergl. Fig. 2, A). Wichtig erscheint uns vor Allem noch ein Befund, der wiederholt erhoben und auf der Abbildung Fig. 2, B dargestellt ist. Wir finden hier eine der oben erwähnten pigmenthaltigen Gliazellen mit deformirtem Kern, der rechte untere Theil ist dicht neben dem mehr links gelegenen Gliakern stark eingebuchtet and schneidet mit einer scharf hervortretenden Begrenzungslinie gegen einen ovalen Hohlraum ab, dessen äussere Grenze durch die dunkler gefärbte Grundsubstanz gebildet wird und in dem ein Lymphocyt liegt. Es könnte sich ja hier um einen zufälligen Befund eines durchwanderten Leukocyten in einer an, eine Gliazelle angrenzenden Masche des ödematösen Grundgewebes handeln; mit Rücksicht auf das wiederholte Vorkommen dieser Verhältnisse und der unten zu erwähnenden Befunde bei den Thierexperimenten scheint uns auch eine andere Deutung zulässig, ja vielleicht gar wahrscheinlicher, auf die wir unten zurückkommen werden.

Aus den. Resultaten der mikroskopischen Durchforschung des übrigen Gehirns, auf die wir hier nicht in extenso eingehen wollen, ist nur noch hervorzuheben, dass sich deutliche Zelldegenerationen, die sich namentlich schön an den Riesenpyramiden darstellen, und diffuser Faserschwund vorfindet. Für die vorliegenden Untersuchungen erscheint jedoch noch wichtig, dass die perivasculären Räume sehr weit, jedoch vollständig frei von 
Blut sind, ebenso sind die pericellulären Räume stark ${ }^{*}$ ausgeweitet und einzelne Pyramidenzellen von den in diesen Räumen angehäuften Lymphocyten geradezu an die Wand gepresst, es finden'sich bis zu 8 Lymphocyten in einem pericellularen Raum. Die Abbildung Fig. 3 veranschaulicht einen weniger extremen Fall, doch finden sich immerhin 4 Lymphocyten in diesem pericellulären Lymphraum. Dies Vorkommen von Lymphocyten in dem ausgeweiteten pericellulären Raum erhärtet die Annahme dieses Raumes als eines präformirten Gebildes und lässt eine Deutung als einfache Schrumpfungserscheinung als irrig erkennen, es mag aber immerhin zugegeben werden, dass die pericellulären Räume durch die Härtung, namentlich bei stark ödematösem Gewebe, vergrössert erscheinen. Eine schon von Meynert beschriebene Thatsache, dass bei starkem Gehirnödem die Protoplasmafortsätze der Pyramidenzellen auffallend weit verfolgt werden können, konnte auch hier festgestellt werden, und einzelne Zellbilder erinnern direct an die mit der Golgi'schen Methode erhobenen Befunde. Obwohl ja Cajal alle Ergebnisse der Golgi-Methode mit Hülfe der Methode der Methylenblauinjection bestätigen konnte, so glauben wir doch, es sei nicht überflüssig, hier 2 Abbildungen solcher einfach mit Thionin gefärbten Zellen zu geben (vergl. Fig. 4 und 5), da sie auch beim erwachsenen Menschen die typische Verzweigung der Protoplasmafortsätze, ihre freie Endigung und die unebene, ja rauhe Beschaffenheit derselben ganz wie an Golgi-Präparaten erkennen lassen. Es sind hier 2 Zellen, die aus der Schicht der grossen Pyramidenzellen der linken Fissura calcarina stammen. Wir glauben, die Berücksichtigung dieses Befundes mit der Bedeutung desselben entschuldigen zu können, obwohl es ja eigentlich streng genommen hier nur insofern erwähnt zu werden verdient, als Rossbach und Sehrwald mit ihrer Methode ähnliche Bilder erhielten, nur in anderer Weise deuteten. Sie zeigen lediglich eine Rückwirkung der Lymphstauung auf die Struktur der Nervenzelle, auf deren feinere Details aber nicht eingegangen werden kann.

Der Befund der blutpigmenthaltigen Gliazellen und Gliafäden scheint uns geeignet, die viel discutirte Frage über die Lymphcirculation in der Grosshirnrinde der Lösung näher zu bringen. Bekanntlich stehen sich, wie schon oben er- 
wähnt, in dieser Frage die Ansichten noch schroff gegenüber. Gehen wir bier auf die in Frage 'kommenden Ansichten noch etwas näher ein, so nimmt $S c h w a l b e^{1}$ ) an, dass 2 durchaus getrennte Hohlraumsysteme existiren, von denen das aus intraadventitiellem Raum und Subarachnoidalraum u. s. w. bestehende System allein als Lymphgefässsystem der Hirnrinde angesprochen werden darf, während das His'sche - extraadventitiell-pericellular-epicerebrale - Hohlraumsystem nicht als ein Lymphgefässsystem angesehen werden könne, da es nirgends mit dem unzweifelhaft lymphatischen ersten Hohlraumsystem oder wirklichen Lymphgefässen eine Communication besitze. Dieser Grund wird aber sofort hinfällig, wenn wir die von $\mathrm{His}$ entdeckte Thatsache berücksichtigen, dass die Arnold'schen Lymphgefässe der Pia durch Injection in die perivasculären Lymphräume gefüllt werden können, die His'schen Räume communiciren also mit unzweifelhaften Lymphgefässen, und wir nehmen am besten, wie dies schon oben hervorgehoben wurde; mit 0 bersteiner ${ }^{2}$ ) und Anderen 2 ächte, aber vollständig getrennte Lymphgefässsysteme an. Im Gegensatz zu der Auffassung Schwalbe's verficht Gierke ${ }^{3}$ ) den Standpunkt, dass die Blutgefässe stets - von den Capillaren abgesehen - von "perivasculären ${ }^{64}$ ) Räumen umgeben sind ${ }^{5}$ ).

1) in Hoffmann's Anatomie. S. 785 .

2) Obersteiner, Anleitung beim Studium des Baues der nervösen Centralorgane. Leipzig und Wien 1896. S. 188.

3) Warum Gierke die Capillaren ausnimmt, ist nicht ganz verständlich, da dieselben mit geringen Ausnabmen - nur die kleinsten Calibers (vergl. Fig. 10) - deutlich von extraadventitiellen Spalträumen umgeben sind. Vergl. Bins wanger, Die pathologische Histologie der Grosshirnrindenerkrankung bei der allgemeinen progressiven Paralyse. Fig. 2. 5.

4) Es herrscht die grösste Verwirrung bezüglich der Nomenclatur dieser beiden grundsätzlich verschiedenen Lymph-, bezw. Saftbahnsysteme. Um nur ein Beispiel unter vielen herauszugreifen, so nennt Gierke die äusseren, zwischen Adventitia und Stätzsubstanz gelegenen Spalten „perivasculäre“ Räume; während Schwalbe dieselbe Bezeichnung für die zwisehen Muscularis und Adventitia gelegenen Lymphbahnen in Anspruch nimmt. Man sollte immer nur von intra- und extraadventitiellen oder intra- und extravasculärea Lywph-, bezw. Saftbahnen sprechen, wie dies oben bereits geschehen ist.

5) Gierke, Die Stützsubstanz des Centralnervensystems. Arehiv für mikroskop. Anatomie. Bd. 26. 1886. 
Die extraadventitiellen Räume sind gegen das Blutgefäss absolut abgeschlossen, während sie nach aussen nur durch die Hirnsubstanz gebildet werden und hier durch Lymphspalten mit dem Hirngewebe überall in Verbindung stehen. Die Mündungen der Saftlücken halten sich gern an die Gliazellen oder deren Ausläufer, welche aus der Hirnsubstanz in die extraadventitiellen Räume eintreten, um durch sie hindurch zur Endotheladventitia zu gelangen. Die Stützsubstanz bildet auf diese Weise die Saftbahnen oder Lymphwege „für die aus den Nervenelementen, besonders aus den Nervenzellen bei ihrer Thätigkeit so ungemein reichlich ausgeschiedene Lymphe. Die Lymphlücken und pericellulären Räume, die extraadventitiellẹn Räume, die oberflächlichen Sammelräume zwischen Gliahülle und Pia mater sind Abschnitte dieses Saftkanalsystems." Das Vorhandensein der intraadventitiellen Lymphräume bestreitet Gierke. Die schon oben erwähnte Auffassung Obersteiner's nimmt eine eigene Begrenzungsmembran des extraadventitiellen und auch des pericellulären Raumes an. Aus der überreichen Literatur erwähnen wir nur noch die Ansicht, die Bevan Lew is ${ }^{1}$ ) in seinem text book of mental diseases, p. 80 und in einer besonderen Arbeit auseinandergesetzt hat. Nach ihm stellen beide Hohlraumsysteme Lymphgefässsysteme dar, und zwar soll der intraadventitielle und extraadventitielle Lymphraum der Gefässe mit einander communiciren. Ausser diesen Lymphräumen soll ein eigenes "Lymphconnectivsystem" der Hirnrinde bestehen, das durch die grossen, häufig mehrkernigen, stets mit einem Gefässfortsatz versehenen Gliazellen, die sich namentlich in der Molecularschicht häufig finden, dargestellt wird. Diese Zellen bilden ein zwischen den Lymphräumen benachbarter Lymphgefässe gelegenes, sogenanntes intervasculäres Lymphsystem. Auf die Bedeutung dieser grossen, in ihrem Bau von den gewöhnlichen Gliazellen abweichenden Elemente als dem Lymphgefässsystem zugeordnete Glieder hat zuerst Meynert aufmerksam gemacht, der sie bei Lymphstauungen im Gehirn, so namentlich auch bei tuberculösen Tumoren der Halslymphdrüsen, die den Lymphabfluss aus der

1) Bevan Lewis, A text book of mental diseases with special neference to the pathological aspects of insanity. London 1889 . p. 80 . 
Schädelhöhle behindern, vergrössert. fand. Auf die Untersuchungen Rossbach's und Sehrwald's ') einzugehen, verzichten wir, da dieselben nicht abgeschlossen sind und von späteren Untersuchern nicht bestätigt werden konnten.

In der von dem einen von uns an dem oben erwähnten Orte gegebenen Darstellung dieser Verhältnisse, der auch die vorstehende Literaturübersicht grösstentheils entnommen ist, ist auf Grund eigener Untersuchungen die Existenz der intra- und extraadventitiellen Saftbahnsysteme bekräftigt worden. Das Studium feiner Rindenschnitte, welche pathologischen und ödematös gestauten Gehirnen entstammen, lassen erkennen, dass schmale Gewebsspalten zwischen den die Capillaren umgebenden Hohlräumen gelegen sind, und auch an den pericellularen Räumen hinziehen. Der Verlauf dieser Lymphspalten wird kenntlich gemacht durch stäbchen- oder spindelförmige blasse Kerne (Hämatoxylinpräparat), und ähnliche Zellbekleidung besitzen die pericellulären Räume, wie dies auch Friedmann klar ausgesprochen hat. Kronthal ${ }^{2}$ ) hat mittelst eines eigenen Verfahrens kleinste, meist geradlinig verlaufende Capillargefässe beschrieben, welche eine ungemein zarte, kerntragende Wandung besitzen. Das Lumen dieser Gefässe ist so eng (2,5 $\mu)$, dass weder farblose, noch weisse Blutkörperchen dieselben passiren können; es fanden sich auch thatsächlich keine körperlichen Elemente in denselben. Diese Untersuchungen bringen zum ersten Male die Ansicht zur Geltung, dass auch innerhalb der Hirnrinde geschlossene, mit eigener Wandung versehene Lymphbahnen existiren. In der oben erwähnten Monographie konnten diese Befunde von Kronthal bestätigt werden; es ist dort auch darauf hingewiesen worden, dass sie sich von den übrigen Capillaren auch dadurch unterscheiden, dass ihre Abzweigungen fast rechtwinklig abgehen und das gleiche Lumen wie das Stammgefäss behalten. Es ist übrigens wahrscheinlich, dass Riedel ${ }^{3}$ ) schon früherhin diese wohl als Lymphoapillaren anzusprechenden

1) Rossbach und Sehrwald, Ueber die Lymphwege des Gehirns. Centralbl. für die med. Wissensch. 188.8.

2) Kronthal, Lymphcapillaren im Gehirn. Neurolog. Centralbl: 1878. S. 679.

3) Riedel, Archiv für mikroskop. Anatomie. 1875. Bd. XI. S, 273. 
Gefässchen aufgefunden hat. Er erwähnt feine, sehr dünne Röhren, die kein axiales Blutgefäss enthalten und die intraadventitiellen Lymphräume benachbarter Blutgefässe verbinden.

Die oben mitgetheilten Befunde lehren erstens, dass in die subarachnoidalen Räume ergossenes Blut als Blutpigment einen Weg bis in die Gliazellen der Molecularschicht gefunden hat; zweitens zeigte sich, dass diese Einwanderung ein activer, durch den Saftstrom vermittelter Vorgang ist, indem nicht alle, sondern nur bestimmte Gliazellen von ihr betroffen werden, während andere, zwischen diesen liegende, von der Einwanderung und auch den Erscheinungen der Lymphstauung vollständig frei bleiben. Dies Moment zeigt klar, dass unter dem Sammelnamen der Gliazelle verschiedene Elemente zusammengefasst werden, wie dies schon Bevan Lewis und Andriezen ausgesprochen haben und dass nur bestimmte, morphologisch ziemlich gut differenzirte Gliazellen es sind, die der Saftcirculation dienen. Der oben besonders hervorgehobene Befund einer einen Hohlraum umschliessenden Gliazelle (Fig. 2 B) lässt die Deutung zu, dass wir es hier mit dem Querschnitt eines in Folge der Staung erweiterten Lymphkanals zu thun haben, welcher, wenn wir einen Schritt weiter gehen wollen, einen jener Riedel-Kronthal'schen Sammelgefässe repräsentirt. Drittens demonstrirt der weitere Befund von Blutpigmenthäufchen in der Umgebung der Blutheerde, insbesondere in der Tiefe der Furchen in der intraadventitiellen Scheide deutlich den bekannten Zusammenhang mit dem subarachnoidalen Lymphraum und die Existenz des von einigen Autoren immer noch angezweifelten intraadventitiellen Lymphraumes. Im Gegensatz zu diesem Befund und ganz entsprechend den Mittheilungen früherer Beobachter konnte viertens in dem extraadventitiellen, sehr erweiterten Lymphraum entweder kein Blutpigment nachgewiesen werden, oder es fand sich nur in dem Leib zweifelloser Leukocyten. Nur an einer Stelle des Temporallappens der linken Seite fand sich der extraadventitielle Lymphraum mit intacten rothen Blutkörperchen angefüllt; doch hier hạdelte es sich höchstwahrscheinlich um eine Gefässzerroissung. Der wenig entwickelte epicerebrale Raum wurde ebenfalls stets frei von Blutkörperchen und Blutpigment gefunden.

Wenn wir uns nochmals die Befunde im Zusammenhang 
vergegenwärtigen, so war das in den Ventrikel ergossene Blut in die subarochnoidalen Räume, von $d a$ in die intraadventitiellen Lymphspalten und auf der anderen Seite in die Gliazellen der Molecularschicht gelangt. Das weite Lymphgefässsystem der Rinde, der pericelluläre Raum, die extraadventitielle Lymphscheide und die epicerebralen Räume erwiesen sich frei von Blutpigment, zeigten jedoch eine deutliche Staung mit Ausnahme des durch den paralytischen Prozess an der Ausweitung gehinderten epicerebralen Raumes. Neu und wichtig erscheint hieran nur, dass die Gliazellen der Molecularschicht mit ihren Fortsätzen bis an den subarachnoidalen Raum reichen und thatsächlich ein Lymphconnectiv-System im Sinne Bevan Lewis darzustellen scheinen und zweitens dass eine Verlegung der intraadventitiellen subarachnoidalen Lymphräume \$eine Drucksteigerung in dem anderen - dem extraadventitiellen - pericellulären Saftbahnsystem zu Folge hat.

Weil über die Verbreitungswege von den subarachnoidalen Räumen aus eine völlig genügende Aufklärung durch diese pathologischen Befunde nicht erreicht war, suchten wir auf dem Wege des Experiments der Lösung dieser Frage etwas näher zu kommen. Wir injicirten einem einjährigen Hunde mittelst einer Pravaz'schen Spritze ganz langsam unter gleichmässig schwachem Druck $0,5 \mathrm{ccm}$ einer Verreibung des gewöhnlichen Carmins in Oleum olivarum in den Subarachnoidalraum. Die Einstichstelle befand sich im Sulc. lateralis des Occipitallappens der rechten Seite; es war wegen der grösseren Ausdehnung des subarachnoidalen Raumes zwischen der Höhe zweier Windungen eine solche Stelle gewählt worden, da an anderen Stellen eine Verletzung der Hirnsubstanz selbst fast unvermeidlich ist bei dem fortwährenden pulsatorischen Schwanken des Grosshirns. Nach 43 Stunden wurde das Thier, das durch den operativen Eingriff sich in keiner Weise geschädigt zeigte, getödtet. Das Gehirn wurde in 10 pCt. Formalin und Alkohol gehärtet. Schon bei makroskopischer Betrachtung fand sich fast in ganzer Ausdehnung der rechten Hemisphäre eine rothe Verfärbung der Hirnoberfläche, welche über den Furchen ihre grösste Intensität und Ausdehnung aufwies. An den senkrecht auf die 
Hirnoberfläche geführten Schnitten (Einbettung in Paraffin nach der üblichen Methode) konnte schon ohne Färbung und bei schwacher Vergrösserung nachgewiesen werden, dass die Carminmischung thatsächlich in dem subarachnoidalen Raum lag und bis in die Tiefen der Furchen eingedrungen war. Die Carminpartikel liessen sich an den ungefärbten Schnitten mit grösster Deutlichkeit längs der in die Rinde einstrahlenden Gefässe bis an die Grenze des Marklagers hin verfolgen und verliehen der Rinde ein eigenartiges rothgestreiftes Aussehen. Genaueres über die Lagerung dieser Carminpartikel liess sich an gefärbten Schnitten und mit stärkerer Vergrösserung feststellen. Die Färbung der Präparate bot erhobliche Schwierigkeiten dar, indem sich sowohl bei der Thionin-, als auch bei der Methylenblaufärbung die Carminpartikel mitfärbten und nicht mehr deutlich als solche erkannt werden konnten. Dagegen gab die gewöhnliche Färbung mit Hämatoxylin, und besonders die Hämatoxylin-Eosin-Färbung befriedigende und sehr klare Resultate.

Es konnte festgestellt werden, dass der Farbstoff von den subarachnoidalen Räumen aus nach zwei Hauptrichtungen verschleppt war, wenn wir hier von dem sehr störenden und manche Klippe für die Deutung der Resultate darbietenden Transport durch wandernde Leukocyten, der im reichen Maasse stattgefunden hatte, absehen. Die Carminpartikelchen waren einerseits in die intraadventitiellen Lymphräume und zweitens in die Gliazellen der oberflächlicheren Schicht des Stratum moleculare gelangt. In beiden fand sich feinkörniges Carmin, in den intraadventitiellen Räumen fanden sich jedoch auch earminhaltige Leukocyten. Die Carminkörnchen, die in die Gliazellen eingedrungen waren, waren auch meist in der Umgebung des Kerns, der keine Deformationen erkennen liess, dichter angehäuft, einzelne Carminkörnchen fanden sich auch streifenförmig in den Gliafäden, welche von den Zellen abgingen. Alle diese Verhältnisse waren an den obersten Gliazellen der Gliahülle besonders deutlich. In der Umgebung der Gliazellen fanden sich zahlreiche Leukocyten, meist mit Carminkörnchen im Leibe. Das in Fig. 8 dargestellte Präparat zeigt die in Frage kommenden Verhältnisse in übersichtlicher Weise. Der erweiterte, von Gliafäden durchzogene, der Pia-Unterfläche anliegende Epicerebralraum stellt hier ein 
vollständiges Maschensystem dar. Zwei Gliazellen, deren Ausläufer den epicerebralen Raum durchsetzen, enthalten reichliche Carminkörnchen, desgleichen eine tiefe, in der Molecularschicht gelegene Zelle, während die grossen Piazellen und eine vierte Gliazelle des Stratum moleculare frei von Carmin erscheint. Dass es sich hier um wirkliche Gliazellen und nicht um die bekannte, in der Pathologie der Zelle schon so oft verhängnissvoll gewordene Verwechselung eines Leukocyten mit einer fixen Bindegewebszelle handelt, scheint uns nach dem Bau der Kerne der Zellen, der von ihnen abgehenden Fäden u. s. w., zweifellos. •

Die Fig. 7, welche den Längsschnitt eines Rindengefässes zur Anschauung bringt, lässt deutlich die kernhaltige Endotheladventitia, die durch feine, die extraadventitiellen Räume überbrückende Fäden mit der Hirnsubstanz zusammenhängt, und der intraadventiell gelagerte, oben freie, unten wohl einem Leukocyten angehörige Carmin erkennen. Neben einzelnen eigenthümlichen, an den Bau der Gliazellen erinnernden, aber im Gegensatz zu diesen mit einem scharf begrenzten Zellleib versehenen Zellen des Stratum moleculare der Rinde, finden sich in einem Hohlraum gelagerte klumpige Massen von Carmin. Diese Befunde, welche Fig. 9 darstellt, erinnern an die analogen Befunde beim Menschen (Fig. 2, B) und dürften wohl ähnlich zu deuten sein; wobei jedoch ausdrücklich hervorgehoben werden soll, dass insofern eine Differenz besteht, indem bei dem Hund aus dem subarachnoidalen Raum diese klumpigen Massen von Carmin nur direct in diese vermeintlichen Lymphgefässe gelangt sein können, während bei der subarachnoidalen Blutung diese Hohlräume frei von Blutpigment gefunden werden.

Eine Füllung des epicerebralen Raumes konnte selbst an der Eintrittsstelle der Gefässe nicht nachgewiesen werden, wohl lagen einzelne Lenkocyten in diesem Raum, die Carminpartikelchen enthielten, doch waren diese Hohlräume selbst frei von solchen. Die einzige Eintrittsstelle von dem subarachnoidalen Raum in die Hirnsubstanz selbst stellen die Gliafäden dar, die den Epicerebralraum durchsetzen und lan der unteren Fläche der Pia anhaften. Hier fanden sich, wenn auch nur an einzelnen Stellen, deutliche Reihen perlschnurartig angeordneter Carminkörnchen. Die extraadventitiellen Räume der Rindengefässe 
waren gleichfalls meistens frei von Carmin und nur in der Umgebung von Kernen der Endothelialadventitia fanden sich vereinzelte Körnchen. Eine fortlaufende Reihe von Körnchen längs der Gliafäden, welche zu diesen Kernen führen, waren nirgends auffindbar. Auch in den pericellulären Räumen fand sich nirgends Carmin, wohl aber in den unvermeidlichen Leukocyten. Die Gliazellen der tieferen Theile des Stratum moleculare waren gleichfalls vollständig frei von Carmin.

Erwähnenswerth erscheint ferner, dass der epicerebrale Raum sehr stark, weniger der extraadventitielle und der pericelluläre Raum kaum gegenüber den normalen Verhältnissen erweitert ist. Einige Bedeutung kommt vielleicht noch einem Befund zu, den das Uebersichtsbild Fig. 6 in klarer Weise zum Ausdruck bringt; man sieht hier umschriebene, meist rundlich begrenzte Hohlräume, die eine deutliche Wand darbieten und unwillkürlich fallen dem Untersucher diese Gebilde auf. Auch uns waren sie gleich bei der ersten Untersuchung aufgefallen, wir vermochten jedoch diese Gebilde nicht zu deuten; erst allmählich entwickelte sich bei uns die Ueberzeugung, dass wir es hier mit enorm ausgedehnten Lymphgefässen zu thun haben müssten; die in der Pia verlaufeu, aber nicht mit dem Subarachnoidalraum communiciren, denn man findet sie stets frei von Carmin. Wir werden wobl hier die von Arnold entdeckten Lymphgefässe der Pia vor uns haben, die mit dem Epicerebralraum communiciren. Die starke Staung in ihnen ist offenbar durch die Verlegung des Subarachnoidalraumes bedingt und entspricht sehr gut der etwas geringeren Staung, die sich im Epicerebralraum findet; diese Ueberlegung bestärkte uns um so mehr in der oben dargelegten Ansicht von der Lymphgefässnatur dieser Gebilde. Analoge Gebilde haben wir beim Menschen nicht gefunden, was uns um so weniger verwundern darf, da wir es mit einer pathologisch veränderten Leptomeninx zu thun hatten und in Folge der chronischen Leptomeningitis der Paralyse ganz abnorme Circulationsverhältnisse in der Pia selbst geschaffen waren.

Der Versuch wurde mit einem etwa $\frac{3}{4}$ Jahre alten 2. Hunde wiederholt, derselbe wurde schon nach 24 Stunden getödtet. Der Befund stimmte in allen Punkten mit dem eben geschilderten überein. Dass die Carminkörnchen durch eine Saftströmung, 
welche von den subarchnoidalen Räumen aus in die Gehirnoberfläche auf den oben genannten Wegen stattfindet, weitergeführt werden, lehrt ein dritter Versuch. Einem einjährigen Hunde wurde in gleicher Weise eine Carminmischung unmittelbar post mortem injicirt. Hier fand sich nun der subarachnoidale Raum mit Carminmassen angefüllt, während die intraadventitiellen Lymphräume - wohl in Folge des schwachen Injectionsdruckes - und die Gliazellen völlig frei von Carminpartikelchen waren.

Wie die vorstehende Schilderung ergiebt, steht der Leichenbefund in völliger Uebereinstimmung mit den Ergebnissen der Injectionsversuche am lebenden Thiere. Es darf daraus geschlossen werden, dass sowohl die Häufungen von Blutpigment als auch die Carmineinlagerungen active Vorgänge fixirt haben, welche mit der Saftströmung zwischen Hirnrinde und Subarachnoidalraum zusammenhängen; freilich kann sowohl der Befund beim Menschen, wie auch der Injectionsversuch nur einen Theil dieser Saftcirculation aufhellen und haben sich die an die experimentelle Untersuchung geknüpften Erwartungen nicht bestätigt. Wir konnten nur die Bedingungen, bei denen unter erhöhtem Druck im Subarachnoidalraum Abflussgebiete nach der Rinde eröffnet werden, studiren. Dass hier der Ausgleich vorzugsweise durch die intraadventitiellen Bahnen vermittelt wird, dass aber auch dem Gliasaftsystem eine Rolle hierbei zukommt, darf als Hauptergebniss unserer Untersuchungen bezeichnet werden; sie bestätigen die von Axel Key und Retzins und Schwalbe gegebene Darstellung des Zusammenhangs der subarachnoidalen und intraadventitiellen Lymphräume, sie zeigen ferner, dass der extraadventitielle-pericellulär-epicerebrale Lymphraum mit dem intraadventitiellen Lymphraumsystem nirgends in offene Communication tritt, und dienen ferner zur Stütze für die Auffassung von Bevan Lewis, dass Antheile der Stützsubstanz (besondere Gliazellen mit ihren Vorläufern) Saftbahnsysteme repräsentiren. Hier scheint vor allen denjenigen Gliazellen, welche einen langen, bis zur Oberfläche reichenden Fortsatz haben, diese Rolle zuzufallen. In welcher Weise der Uebertritt von Flüssigkeit oder festen Partikelchen in diese Gliasaftbahn bewerkstelligt wird, ist auch durch unsere Untersuchungen nicht ganz aufgeklärt, doch 
machen es unsere Befunde sehr wahrscheinlich, dass an den Stellen, an denen sich die Gliaausläufer an die Pịa anheften, Saftlücken vorhanden sind. Es ist ferner durch die Vertheilung des Blutpigments und der Carminmassen wahrseheinlieh gemacht, dass diesen Gliasaftbahnen nur eine territorial-begrenzte Bedeutung zukommt, sie also nur für die Ernährung kleinster Bezirke, welche der nächsten Umgebung dieser Gliazellen angehörig sind, in Betracht kommen. Nach den herrschenden, auch durch die Weigert'sche Glia-Methode bestätigten Ansichten bestehen zwischen den Gliafäden keine Communicationen, sie heften sich aber wohl an Gefässe an, wie dies auch Fig. 10 darstellt, die eine Gehirncapillare im Zusammenhang mit einer benachbarten kernigen Gliazelle zeigt. Das Präparat, welches auch sehr deutlich dies eigenthümliche gabelförmige Umgriffenwerden des Endothelkernes durch den Gefässfortsatz der Gliazelle und den Mangel jeder, intra- und extraadventitieller, Iymphscheide an den feinsten Capillaren demonstrirt, entstammt dem Lobus paracentralis einer an Dementia senilis leidenden und in Folge einer Bronchopneumonie verstorbenen 85jährigen Frau. Schon das Fehlen von Communicationen zwischen den Gliafäden verschiedener Gliazellen bedingt eine enge Beschränkung des Wirkungskreises der einzelnen Gliazelle. Das Blutpigment und die Carminkörnchen müssen also längs einer oder mehrerer Zellfortsätze in jede Zelle gesondert eingedrungen sein, und das beweist, dass selbst verbältnissmässig tief in der Molecularschicht liegende Zellen einen bis an die Pia reichenden Fortsatz besitzen müssen. Von der Weigert'schen Ansicht, welche annimmt, dass beim Erwachsenen die Gliafäden selbständige, nicht mit den Kernen zusammenhängende Gebilde sind, konnten wir uns nicht überzeugen, obwohl wir es mit dem Gehirn eines älteren Mannes zu thun hatten, ja, die fadenförmig angeordneten Carminkörnchen liessen sich ebenso wie die Blutpigmentpartikelchen deutlich in dem reichlich den Kern umgebenden und in Folge der Staung deutlicher hervortretenden Zellplasma verfolgen.

Zuletzt wollen wir noch als ein Ergebniss unserer Untersuchungen erwähnen, dass sie sämmtlich darthun, dass die Verlegung des Subarachnoidalraumes eine Drucksteigerung in dem pericellulär-extraadventitiell-epicerebralen Lymphgefässsystem 
zu Folge hat. Beim Menschen zeigt sich dies besonders deutlich an den extraadventitiellen und namentlich den pericellulären Räumen, beim Hund tritt vor Allem die Staung im Epicerebralraum und in den Arnold'schen Lymphgefässen der Pia hinzu. Wir könnten schon aus diesem Befund, selbst ohne Kenntniss von dem exacten Nachweis der Zusammengehörigkeit aller dieser letztgenannten Lymphräume durch $\mathrm{His}$, auf diese Communication schliessen. Ein Circulationshinderniss bedingt in den dem Hinderniss benachbarten Theilen einer geschlossenen Gefässbahn die ersten Staungserscheinungen. Indem wir nun die verschiedenen Stärken der Lymphstauung, die sich beim Hunde sehr deutlich markirten — das menschliche Gehirn kommt wegen der abnormen Circulations-Verhältnisse der erkrankten Pia weniger in Betracht - müssen wir schliessen, dass die Lymphbewegung - wie dies auch schon His forderte - von den pericellulären Räumen za dem extraadventitiellen und von diesem zu dem epicerebralen Lacunensystem und den Pialymphgefässen stattfindet. Dieses Gefässsystem würde demnach ein wahres Lymphgefässsytem darstellen und den Lymphbahnen anderer Körperorgane an die. Seite zu stellen sein. Das Ventrikelsystem dagegen mit den mit ihm communicirenden subarachnoidalen and intraadventitiellen Lymphspalten besitzt lediglich eine Bedeutung für die Regulirung des hydrostatischen Druckes, kann aber nicht als Lymphgefässsystem im engeren Sinne angesprochen werden.

\section{Erklärung der Abbildungen. Tafe] X-XI.}

Fig. 1. Uebersichtsbild über die Lagerung und Ausdehnung des subarachnoidalen Blutergusses. Der Sclnitt ist senkrecht auf die Verlaufsrichtung der ersten linken Temporalwindung geführt. Hämatoxylinpräparat. Schwache Vergrösserung. A Arachnoidea. SF Subarachnoidealfäden. P Pia. G Gefässe. Gl Gliazellen im Stratum moleculare. B Blutcoagula. L Lenkocyten mit verändertem Blutfarbstoff.

Fig. 2. Aeusserster Theil des Stratum moleculare der ersten linken Temporalwindung bei starker Vergrösserung (Comp.-Oeul. 4 und Apocbrom. Immersion $2,0 \mathrm{~mm}$ ). Man sieht die sich intensiver färbende. Gliahülle und die zahlreichen, mit Blutpigment erfüllten Gliazellen. A gewöhnliche Gliazelle ohne Blutpigment. B Lympheapillare (?). Färbung mit Tbionin-Eosin. Schnittdicke $10 \mu$. 
Fig. 3. Nervenzelle aus der Schicht der grossen Pyramidenzellen der ersten Temporalwindung der linken Seite. Man sieht vier Leukocyten in dem erweiterten pericellulären Raum liegen. Färbung mit ThioninEosin. Sehnittdicke $10 \mu$. Comp.-Ocul. 4, Apochr. Immersion 2,0 mm.

Fig. 4. Solitärzelle aus der Rinde der rechten Fissura calcarina mit weithin sichtbaren, frei endenden Protoplasmafortsätzen. Thioninfärbung. Schnittdicke $5 \mu$. Comp.-0cul. 4, Apocbrom. Immersion $2,0 \mathrm{~mm}$.

Fig. 5. Pathologisch veränderte Zelle aus der Schicht der grossen Pyramide. Zellen der Rinde der rechten Fissura calcarina ebenfalls mit weithin sichtbaren Protoplasmafortsätzen. Thioninfärbung. Schnittdicke 5 u. Comp.-Ocul. 4, Apochr. Immersion $2,0 \mathrm{~mm}$.

Fig. 6. Uebersichtspräparat über die Lagerung der Carminkörnchen bei Hund I (getödtet nach 43 Stunden). Hämatoxylinpräparat bei schwacher Vergrösserung. L die Arnold'schen Lymphgefässe der Pia (?).

Fig. 7. Rindengefäss von Hund I. Färbung mit Hämatoxylin-Eosin. Comp.0cul. 4, Apochr. Immersion. $2,0 \mathrm{~mm}$. Schnittdicke $10 \%$. Lu Lumen des Gefässes. In Intima. Ad Adventitia (die sich nicht überall deutlich abhebt). IL intraaadventitieller Lymphraum. EL extraadventitieller Lymphranm. Man sieht deutlich die den extraadventitiellen Lymphraum durchziehenden Fäden. Oben liegen in der Hirnsubstanz 2 Leukocyten.

Fig. 8. Oberster Rindentheil einer Windung von Hund I. Färbung mit Eämatoxylin-Eosin. Comp.-Ocul, 4, Apochr. Immersion 2,0 mm. Schnittdicke $10 \mu$. P der unterste Theil der Pia. ER das epicerebrale Lacunensystem. Strat. mol. oberster Theil des Stratum moleculare der Rinde.

Fig. 9. Lymphcapillare der Hirnrinde (?). Stratum moleculare von Hund I. Färbung mit Hämatoxylin. Comp.-Ocul. 4, Apochr. Immersion 2,0 mm. Schnittdicke $10 \mu$.

Fig. 10. Capillare der Hirnrinde des Lobus paracentralis einer 85 jährigen, an Dementia senilis leidenden Frau. Färbung mit ToluidinblauEosin. Comp.-Ocul. 6, Apochr. Immersion 2,0 mm. Schnittdicke $10 \mu$. Die grosse 2 kernige Gliazelle entsendet ausser mehreren auderen einen dicken, gerade nach unten gerichteten Gefässfortsatz, der den Kern der Capillare gabeiförmig umgreift. An der Capillare ist weder ein intra-, noch ein extraadventitieller Lymphraum sichtbar. 